\title{
Efecto del tratamiento anti-helmíntico sobre los niveles de hemoglobina
}

Effect of intestinal antihelmintic administration on hemoglobin levels

Gulani A, et all. BMJ. 2007 May 26;334(7603):1095.

\section{Objetivo}

Evaluar el efecto de la administración rutinaria de drogas antihelmínticas sobre los niveles de hemoglobina.

\section{Fuente de datos}

Bases de datos bibliográficas Medline y Cochrane, resúmenes de conferencias internacionales, y búsqueda manual en libros y citas bibliográficas de las publicaciones identificadas.

\section{Selección de estudios}

Se incluyeron ensayos clínicos controlados (ECC) aleatorizados o cuasi-aleatorizados que hubieran utitilizado agentes antihelmínticos intestinales en el grupo intervención y evaluado como resultado los niveles de hemoglobina.

\section{Extracción de datos}

Los datos fueron extraídos por duplicado por dos investigadores. Fueron incluidos 14 ECC, 11 de ellos realizados sobre población pre-escolar y escolar, incluyendo el resto a mujeres adultas (no embarazadas y embarazadas) y a grupos de diferentes edades. En diez trabajos se utilizó albendazol como droga antihelmíntica, en tres mebendazol y en uno, bephenium. En más de la mitad de los estudios se uso terapia con hierro como co-tratamiento.

\section{Resultados principales}

La Organización Mundial de la Salud (OMS) recomienda niveles mínimos de hemoglobina de hasta $12 \mathrm{~g} / \mathrm{L}$ en adultos y $11 \mathrm{~g} / \mathrm{L}$ en niños. Sobre un total de 7829 pacientes, 4107 recibieron tratamiento antiparasitario y 3722 , placebo. Los valores de hemoglobina aumentaron un promedio de 1,71g/L (IC95\%: 0,70 a 2,73) luego del tratamiento. No se observaron diferencias significativas luego de ajustar por la co-intervención con hierro. El presente meta-análisis estimó que, asumiendo una respuesta promedio, el tratamiento anti-helmítnico rutinario se asociaría a una reducción de 1,1 a 12,4\% de la prevalencia de anemia en adultos y de 4,4 a $21 \%$ en niños, incrementándose el impacto de esta estrategia cuando se considera puntos de corte de hemoglobina más bajos en la definición de esta condición clínica.

\section{Conclusiones}

La administración rutinaria de drogas antihelmínticas da como resultado un mínimo incremento en los valores de hemoglobina $(1,71 \mathrm{~g} / \mathrm{L})$ lo que trasladado a una escala poblacional $y$ teniendo en cuenta la alta prevalencia de las parasitosis intestinales, representaría una pequeña reducción (5 a 10\%) de la prevalencia de anemia.

Palabras clave: parasitosis, anemia, hemoglobina, drogas anti-helmínticas, hierro. Key words: parasitic infections, anemia, hemoglobin, anti-helmintic drugs.

Fuente de financiamiento: Sitaram Bhartia Institute of Science and Research, New Delhi, India (no se involucró en el diseño del estudio ni en la escritura del manuscrito).

\section{Comentario}

Las infecciones parasitarias continúan siendo un importante problema de salud pública, ya que influyen negativamente sobre el estado nutricional de los niños, predisponiéndolos a una mayor prevalencia de anemia, alteraciones en el crecimiento pondo-estatural y trastornos del aprendizaje $e^{1,2,3,4,5,6}$. Cabe destacar que la OMS y el Banco Mundial recomiendan tratar en forma rutinaria con antiparasitarios a los niños que viven en países en vías de desarrollo.

Estudios previos habían demostrado que la adición de tratamiento antiparasitario a la suplementación con hierro se asocia a mayores incrementos de los niveles de hemoglobina; y que por otro lado, la suplementación con hierro como monoterapia es superior a la ausencia de tratamiento ${ }^{7,8,9,10}$. Sin embargo, no se encontró evidencia sustancial a favor de la suplementación con hierro sobre los parámetros antropométri$\cos ^{11}$ y sobre las funciones cognitivas de los niños ${ }^{12,13}$.
El presente meta-análisis intenta, con un muy buen criterio metodológico, documenta que la administración rutinaria de antihelmínticos e independientemente del número de dosis utilizadas, se asociaría a un pequeño incremento en los valores de hemoglobina $(1,71 \mathrm{~g} / \mathrm{L})$. Sin embargo, al hablar de tratamiento rutinario, resulta importante considerar los efectos indeseables de las drogas antiparasitarias (en especial sobre la salud de las personas no infectadas) así como su costo-efectividad.

\section{Conclusiones del comentador}

El tratamiento rutinario con antihelmínticos debe ser considerado en cada zona o país en particular, de acuerdo a su costoefectividad, a la prevalencia de los factores analizados, y al impacto que generaría sobre el crecimiento pondo-estatural y las funciones cognitivas de los niños.

Javier Chiarpenello [ Médico Generalista y de Familia. Médico Endocrinólogo. Hospital Centenario de Rosario. jchiarpenello@ hotmail.com ]

Chiarpenello J. El tratamiento rutinario con antihelmínticos mejora los niveles de hemoglobina. Evid. actual. práct. ambul; 10(5):138, Sep-Oct 2007. Effect of intestinal anthelmintic administration on haemoglobin levels: systematic review of randomised controlled trials. Gulani $A$, et all. BMJ, May 2007; 334: 1095 ; doi:10.1136/bmj.39150.510475.AE. PMID: 17434954. Disponible en URL; http://www.bmj.com/cgi/reprint/334/7603/1095

Referencias

1. Dickson R, et all. Anthelmintic drugs for treating worms in children: effects on growth and cognitive performance. Cochrane Database Syst Rev. 2000;(2):CD000371

2. Dickson R, el al. Anthelmintic drugs for treating worms in children: effects on growth and cognitive performance. Cochrane Database Syst Rev. 2007 Jul 18;(3):CD00037

3. Crompton D, et al. Nutritional impact of intestinal helminthiasis during the human life cycle. Annu Rev Nutr. 2002;22:35-59. Epub 2002 Jan 4.

4. Egger R, et all. Association between intestinal parasitoses and nutritional status in 3-8-year-old children in northeast Thailand. Trop Geogr Med. 1990 Oct;42(4):312-23.

5. Ulukanligil M, et all.Anthropometric status, anaemia and intestinal helminthic infections in shantytown and apartment schoolchildren in the Sanliurfa province of Turkey. Eur J Clin Nutr. 2004 Jul; 58(7):1056-61.

6. Quihui-Cota L, et all. Prevalence and intensity of intestinal parasitic infections in relation to nutritional status in Mexican schoolchildren. Trans R Soc Trop Med Hyg. 2004 Nov;98(11):653-9.

7. Gera T, et all.Effect of iron supplementation on haemoglobin response in children: systematic review of randomised controlled trials. J Pediatr Gastroenterol Nutr. 2007 Apr;44(4):468-86.

8. Gera T, et all. Effect of iron supplementation on physical performance in children and adolescents: systematic review of randomized controlled trials. Indian Pediatr. 2007 Jan;44(1):15-24

9. Taylor M, et all. The effect of different anthelmintic treatment regimens combined with iron supplementation on the nutritional status of schoolchildren in KwaZulu-Natal, South Africa: a randomized controlled trial. Trans R Soc Trop Med Hyg. 2001 Mar-Apr:95(2):211-6.

10. Friis $\mathrm{H}$, et all. Effects on haemoglobin of multi-micronutrient supplementation and multi-helminth chemotherapy: a randomized, controlled trial in Kenyan school children. Eur J Clin Nutr. 2003 Apr:57(4):573-9.

11. Sachdev H, et all. Effect of iron supplementation on physical growth in children: systematic review of randomised controlled trials. Public Health Nutr. 2006 Oct;9(7):904-20.

1. Sachdev $\mathrm{H}$, et all. Efect of iron supplementation on physical grom in

12. Dickson R, et all. Effects of treatment for intestinal helminth infection on growth and cognitive performance in children: systematic review of randomised trials. BMJ. 2000 Jun 24;320(7251):
13. Sachdev H, et all. Effect of iron supplementation on mental and motor development in children: systematic review of randomised controlled trials. Public Health Nutr. 2005 Apr;8(2):117-32. 